\title{
Place de l'histoire des sciences et des techniques dans les enseignements et la recherche universitaires : états des lieux et enjeux
}

\section{Hélène Gispert}

\section{OpenEdition}

\section{Journals}

Édition électronique

URL : http://journals.openedition.org/trema/71

DOI : $10.4000 /$ trema.71

ISSN : 2107-0997

Éditeur

Faculté d'Éducation de l'université de Montpellier

Édition imprimée

Date de publication : 1 octobre 2006

Pagination : 5-10

ISSN : 1167-315X

Référence électronique

Hélène Gispert, « Place de l'histoire des sciences et des techniques dans les enseignements et la recherche universitaires : états des lieux et enjeux », Tréma [En ligne], 26 | 2006, mis en ligne le 04 octobre 2010, consulté le 19 avril 2019. URL : http://journals.openedition.org/trema/71 ; DOI 10.4000/trema.71

Ce document a été généré automatiquement le 19 avril 2019.

Trema 


\title{
Place de l'histoire des sciences et des
} techniques dans les enseignements et la recherche universitaires : états des lieux et enjeux

\author{
Hélène Gispert
}

1 Le titre de cet article est manifestement très ambitieux. Il traduit, certes, une ambition tout à la fois légitime et nécessaire pour tous ceux que l'avenir de l'histoire des sciences et des techniques comme discipline universitaire intéresse, mais je ne suis pas certaine que nous disposions à ce jour des éléments qui me permettent de la tenir.

Une première approche permet de mesurer la place d'une discipline dans les enseignements et la recherche universitaire ; il s'agit de connaître le nombre des enseignants-chercheurs qui relèvent de ce champ, le nombre des équipes qui mènent des recherches dans le domaine en question, ainsi que le nombre des publications, des thèses soutenues ou en cours, le nombre des doctorants engagés dans de telles recherches. Essayons donc.

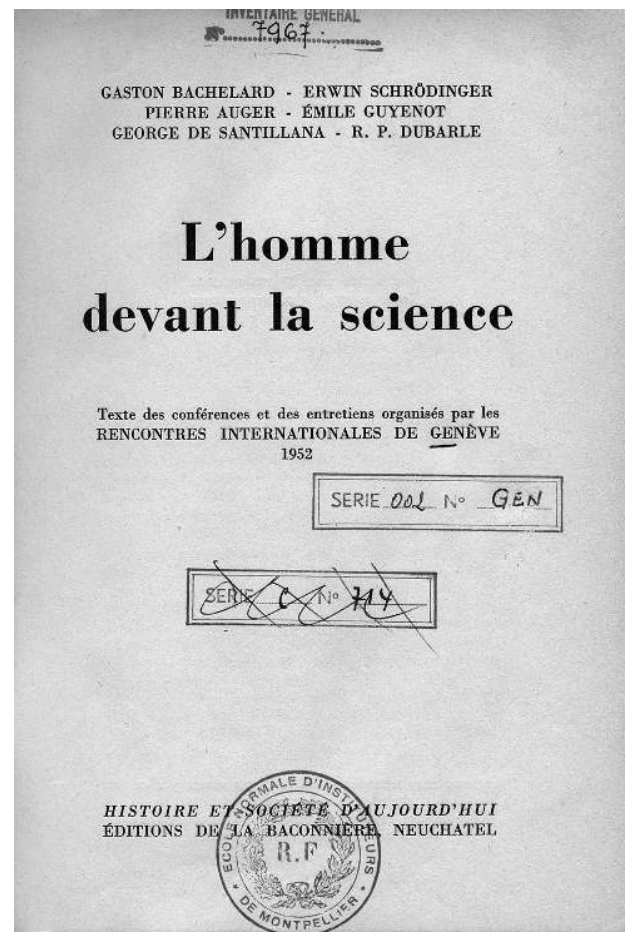

GASTON BACHELARD - ERWIN SCHRO̊DINGER PIERRE AUGER - ÉMILE GUYENOT

\section{L'homme}

devant la science

Texte des conférences et des entretiens organisés par les 1952

SERIE OOL NO GEN HISTOIRE ET SSOCTETE W OUJOURD'HUI 


\section{Quel est le nombre des enseignants-chercheurs ?}

3 Avec cette question, qui pourrait paraître simple, surgit une première complication spécifique à notre domaine de l'histoire des sciences et des techniques. Doit-on les chercher dans la seule section «Epistémologie, histoire des sciences et des techniques » du Conseil national des universités ( $\mathrm{CNU}$ ), c'est-à-dire la section $72^{1}$ ? Ou doit-on prendre en compte des collègues d'autres sections qui recrutent également des historiens ou philosophes des sciences comme la section 17, philosophie, ou la section 22, histoire, voire d'autres encore comme les sections des disciplines d'origine dont, en particulier, la section 25 de mathématiques?

4 Si l'on regarde les postes de la section 72, la seule dont les postes soient « officiellement » labellisés « Histoire des sciences et des techniques », il y avait à la rentrée 2004, soixantedix-sept enseignants chercheurs. Comme élément de comparaison, en 2001-2002, toutes disciplines confondues, on comptait 54500 enseignants-chercheurs ; sur la faculté des sciences d'Orsay, pour donner une comparaison locale, il y a trois enseignants-chercheurs 72 - ce qui est beaucoup pour une faculté de sciences - pour plus de 100 en mathématiques.

5 La section 72 est ainsi une des plus petites, mais il est intéressant de noter que depuis sa création, au milieu des années 1980, elle connaît une croissante importante, le nombre d'enseignants chercheurs ayant été multiplié par trois entre 1996 et 2004 (voir annexe 1).

6 Mais, nouvelle spécificité de l'histoire des sciences et des techniques (HST) dans le champ universitaire, ces postes d'enseignants-chercheurs ne correspondent qu'à une facette de la situation, la réalité des postes ne recouvrant pas la réalité des enseignements. J'en veux pour preuve l'enquête menée en $2003^{2}$ à l'initiative de Marta SPRANZI-ZUBER, Amy DAHAN et moi-même sur la réalité des enseignements en HST : une des conclusions manifestes en a été leur dispersion et leur émiettement ; plus de la moitié des collègues qui ont répondu faisait moins de 50 heures d'enseignement en HST, beaucoup d'entre eux enseignant autour de 20 à 25 heures incluses dans un service consacré à une autre discipline. Une autre enquête, menée par Laurent ROLLET en 2002 sur la ville de Nancy ${ }^{3}$, estimait à 1880 heures TD les enseignements dispensés en «études sur les sciences », soit un domaine plus vaste que l'HST, dans les établissements d'enseignement supérieur, c'est-à-dire les universités mais, en grande partie, les différentes écoles. Le nombre d'heures enseignées, si elles n'avaient pas été assurées pour l'essentiel par des collègues de tout horizon et en partie par vacations, aurait nécessité la création de huit postes d'enseignants-chercheurs. Dernier élément, instructif et préoccupant, l'enseignement en HST est couramment délivré par des collègues expérimentés mais sous forme de vacations comme le montre le profil des personnes candidates à la qualification, et qualifiées, en section 72 .

7 Je pense qu'il ne faut pas déconnecter la question de la place de l'HST de celle de ses enjeux et il nous faut ici pointer deux enjeux importants.

8 La section 72 connait certes une forte dynamique de croissance, mais elle demeure une petite section ce qui a des conséquences dommageables. Ses enseignants-chercheurs - et plus souvent encore les autres collègues qui enseignent - se trouvent isolés, en particulier en faculté des sciences, en IUFM, en écoles d'ingénieurs. Ils ne peuvent ainsi bénéficier de la dynamique d'un département d'enseignement, d'une équipe locale de 
recherche alors que la plupart de nos enseignements sont à créer, à inventer et pas seulement à dispenser, et cela en lien à la recherche, ce qui doit être la caractéristique d'un enseignement universitaire.

9 Autre enjeu lié à la question de la place de l'HST et qui ressort des deux enquêtes citées, celui de la nature des cursus en HST. Il apparaît en effet que les filières les plus consommatrices d'enseignements en HST sont la médecine (à cause du concours et de la place qu'y ont les sciences humaines), les écoles d'ingénieurs et, en faculté des sciences, les ex-DEUG ou L1 et L2. Cette situation induit une demande très instrumentalisée ou très élémentaire, voire rudimentaire en volume horaire, devant de gros amphis, qui aboutit à un saupoudrage et qui le plus souvent ne permet pas ce lien nécessaire de l'enseignement et de la recherche. Quant aux enseignements plus spécialisés, une autre traduction de cette réalité est le petit nombre de masters et d'écoles doctorales en HPS, et plus encore en faculté des sciences. Se trouve ainsi posée la question des finalités des enseignements en HST à l'université, question sur laquelle je reviendrai.

Les enquêtes ci-dessus le montrent, des besoins d'enseignements en HST existent, plus encore des demandes explicites sont aujourd'hui satisfaites dans des conditions de dispersion et de précarité pré-occupantes. Mais a-t-on les ressources pour changer la donne et assurer ces besoins en termes de créations de postes ? Les campagnes de qualification de la section 72 permettent de donner une réponse sans ambiguïté à une telle question. Il y a un vivier suffisamment important de collègues qualifiés, souvent expérimentés, pour répondre à toute politique ambitieuse de créations de postes en HST; en effet, de 2000 à 2005 la section 72 du CNU a qualifié 264 collègues aux fonctions de maître de conférences et 70 aux fonctions de professeurs (voir annexe 2).

11 Venons-en à présent à l'aspect recherche et regardons, comme mesure de la dynamique de notre domaine HST, ce qu'il en est des doctorants, des équipes. Une enquête, qui ne prétend pas à l'exhaustivité et qui repose sur les données qui nous ont été fournies, a été menée sur le site de THEUTH (http://theut.univ-rennes1.fr//) à l'initiative de Alain HERREMAN et moi-même.

12 Nous avons recensé au 17 mai 2005212 thèses en cours en épistémologie et histoire des sciences et des techniques. La répartition des doctorants par école doctorale (voir annexe 3) fait apparaître trois lieux forts : l'université de Paris 7 (36 thèses en cours), celle de Paris 1 ( 31 thèses en cours) et celle de Nantes ( 30 thèses en cours), puis les universités de Lille (21 thèses en cours) et Paris 10 (14 thèses en cours). Quant aux équipes de recherche, la liste des équipes ayant participé à la constitution de cette base de données sur les thèses est indiquée en annexe 4.

13 Je voudrais, pour finir, aborder la question signalée précédemment, des finalités de nos enseignements en HST. Le double constat de la petite taille de la section 72 et de l'ampleur du vivier potentiel en épistémologie et histoire des sciences et des techniques ne peut qu'avoir des conséquences manifestes sur les missions de cet enseignement d'HST. Se pose en effet la question - que l'on n'a pas en IUFM - qui a fait largement débat lors de la rencontre de 2003 où ont été présentés et débattus les résultats de l'enquête initiée au plan national mentionnée plus haut : peut-on considérer que notre enseignement dans les universités a principalement à former de futurs historiens des sciences ? Ou, au contraire, que l'histoire des sciences doit y être d'abord une discipline de service? 
14 Les réponses à ces questions ne font pas aujourd'hui l'unanimité. Il pourrait être intéressant que nous les discutions ici, mais je voudrais, dans le cadre de cette discussion apporter deux éléments de réponse personnels. Il me semble tout d'abord qu'il ne serait pas responsable d'ignorer la question, particulièrement aiguë dans notre champ, des perspectives d'emploi qui sont extrêmement réduites en ce qui concerne l'enseignement et la recherche, et de faire de la formation de futurs spécialistes de l'HST l'objectif principal de notre enseignement. Mais ceci ne doit en rien empêcher de se battre pour réclamer, exiger des postes. Un des facteurs de croissance a été la création des postes dits «postes Lecourt »; or, les promesses n'ont pas été entièrement tenues et on nous doit encore une campagne de postes.

Mon deuxième élément de réponse vient de notre expérience sur Orsay et me permettra de préciser ma position sur ce concept de "discipline de service ". Ce concept ne me gêne pas, en ce qui concerne la formation, dans la mesure où, tout en devant nous adapter à des perspectives de formation qui ne sont pas autonomes, nous mettons en œuvre, à ces fins, des contenus et des méthodes nourris, construits à partir d'une réflexion, d'une activité en prise avec la recherche en HST, avec les objets, les idées, les problématiques débattues aujourd'hui en recherche. Il ne s'agit donc aucunement d'en rabattre mais au contraire de créer dans le maximum de centres, à partir des demandes qui nous sont faites, les conditions d'enseignement et de recherche qui nous permettent de proposer des enseignements en HST de qualité. Et, pour conclure, je tiens à dire qu'il est particulièrement intéressant, et possible, de tenir cet objectif en IUFM.

\title{
ANNEXES
}

\section{Annexe 1 - mai 2005}

\section{Postes 72 e section (évaluation)}

Rentrée 2004 :

- 77 enseignants-chercheurs $=55 \mathrm{MCU}-22 \mathrm{PR}$

- (toutes disciplines du CNU, en 2001-2002 : 54500 enseignants-chercheurs ; le ratio MCU/PR étant 2/3-1/3)

\section{Evolution du nombre de postes}

\author{
1996 : 28 enseignants-chercheurs $=15$ MCU-13 PR \\ renouvellement) \\ Février 2001 : premiers postes Lecourt \\ Septembre $2004: 77$ enseignants-chercheurs = 55 MCU-22 PR \\ - de 1996 à 2004 : nbre multiplié par 3 \\ - nbre MCU augmente et nbre PR stable \\ Septembre $2005:$ ? (double affichage des postes)
}

2000 : 58 enseignants-chercheurs $=30$ MCU-28 PR (pb d'interprétation $:$ créations ou 


\section{Annexe 2 - mai 2005}

Vivier 72e section

\section{Depuis 1992 (sauf 1996 et 1997)}

La section 72e du CNU a qualifié

- 343 candidats aux fonctions de MCU= 312 personnes

- 100 candidats aux fonctions de PR (même remarque)

\section{Périodisation}

De 1992 à 1999 (sauf 1996 et 1997)

- 93 MCU et 30 PR qualifiés c.a.d. MCU : 18/an et PR : 6/an

De 2000 à 2005

- 244 MCU et 70 PR qualifiés c.a.d. MCU : 40/an et PR : 11/an

THEUTH (http://theut.univ-rennes1.fr)

\section{Annexe 3 - mai 2005}

\section{Thèses en cours (212 au 17 mai 2005)}

\section{Epistémologie et Histoire des Sciences}

- Université Lille $3: 21$ thèses en cours

- Université Paris $1: 31$ thèses en cours

- Université Paris $6: 4$ thèses en cours

- Université Paris $7: 36$ thèses en cours

- Université Paris $8: 1$ thèse en cours

- Université Paris $10: 14$ thèses en cours

- Université Paris $11: 1$ thèse en cours

- Université Nancy $2: 1$ thèse en cours (sur leur site web : 19 annoncées)

- Université Nantes : 30 thèses en cours

- Université Strasbourg $1: 5$ thèses en cours

- Université Lyon 1 et Lyon $2: 3$ thèses en cours

- Université Bordeaux $1: 2$ thèses en cours

- CNAM/CDHT : 21 thèses en cours

- EHESS : 10 thèses en cours (biais)

\section{Annexe 4 - mai 2005}

\section{THEUTH Epistémologie et Histoire des Sciences}

Structures de recherches universitaires (UMR et EA) ayant participé à la constitution de la base de données sur les thèses.

Le site de l'IRIST (Université Strasbourg 1) 
Le Centre d'histoire des sciences et des philosophies arabes et médiévales CNRS/

Université Paris 7/ÉPHÉ (UMR 7062)

Le Centre d'histoire et de philosophie des sciences (Université Paris 10)

L'équipe d'Histoire des sciences mathématiques de l'IMJ

(Université Paris 6)

L'IPHST (Université Paris 1)

L'UMR 8519 "Savoirs et textes" (Université Lille 3)

Le Centre François Viète (Université Nantes)

Le CNAM/CDHT

Le Centre Alexandre Koyré (UMR EHESS-MNHN-CNRS)

Le REHSEIS (UMR Paris 7-CNRS)

Le GHDSO (Université Paris 11)

L'UMR Archives Henri Poincaré (Université Nancy 2)

EPISTEME (Université Bordeaux 1)

\section{NOTES}

1. A titre d'information je donne ici un extrait des critères de qualification établis par la section 72 qui concerne les domaines de recherche couverts par la section (le document complet se trouve sur le site du CNU ou le site de Theuth - Epistémologie et histoire des sciences, actuellement à l'adresse : http://theuth.univ-rennes1.fr.) : “Un consensus s'est dégagé pour accepter les candidatures portant sur la logique, l'épistémologie, la philosophie des sciences (analytique ou continentale), l'histoire des sciences (logique, mathématiques, sciences de la nature, sciences humaines et sciences sociales), l'histoire de la médecine et l'histoire des techniques, l'histoire des institutions scientifiques, médicales et techniques, les études sociales ou culturelles des sciences et des techniques, les études de la diffusion (par exemple, la muséologie) et de l'impact des sciences, l'éthique des sciences, des techniques et de la médecine. L'éventail est suffisamment large pour qu'on exige des candidats une orientation prononcée dans l'un de ces domaines, manifestée sous forme de publications dans des revues spécialisées et d'engagement dans une équipe de recherche."

2. Pour les objectifs de cette enquête et le texte du questionnaire, voir le site de Theuth Epistémologie et histoire des sciences. Les résultats de l'enquête ont été présentés oralement lors d'une journée nationale en juin 2003 mais n'ont pas fait l'objet de publication.

3. Le rapport de Laurent Rollet est accessible sur le site Theuth. 


\title{
RÉSUMÉS
}

Dans un premier temps nous cherchons à donner une estimation quantitative de la place de l'HST dans les dix dernières années : nombre d'enseignants chercheurs en histoire des sciences et des techniques, nombre et types d'enseignements dispensés, nombre de thèses en cours. Cela nous conduit à examiner quelques enjeux de l'enseignement de l'HST et de ses finalités, son rôle et sa qualité comme discipline de service ou discipline autonome, ses liens avec la recherche.

At first we try to give a quantitative estimation of the place of the history of sciences in the last ten years: number of teachers researchers in history of the sciences and the techniques, the number and the types of exempt educations, number of current these. It leads us to examine the stakes in the education of the EHST of its ends, its role and its quality as discipline of service or autonomous discipline, its links with the research.

\section{INDEX}

Mots-clés : finalité, histoire des sciences, poste, recherche

\author{
AUTEUR \\ HÉLĖNE GISPERT \\ Groupe d'histoire et de diffusion des sciences d'Orsay GHDSO Université Paris Sud \\ helene.gispert@ghdso.u-psud.fr
}

